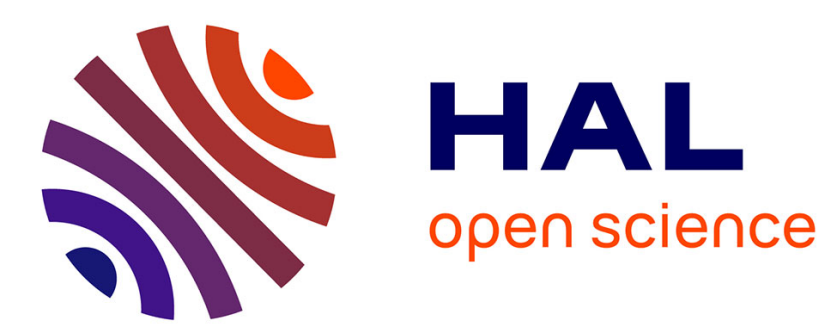

\title{
Évaluer la douleur en rhumatologie - comment faire mieux ? Revue de la littérature
}

Florian Bailly, Bruno Fautrel, Laure Gossec

\section{To cite this version:}

Florian Bailly, Bruno Fautrel, Laure Gossec. Évaluer la douleur en rhumatologie - comment faire mieux ? Revue de la littérature. Revue du Rhumatisme, 2016, 83 (2), pp.105-109. 10.1016/j.rhum.2015.07.013 . hal-01288228

\section{HAL Id: hal-01288228 https://hal.sorbonne-universite.fr/hal-01288228}

Submitted on 14 Mar 2016

HAL is a multi-disciplinary open access archive for the deposit and dissemination of scientific research documents, whether they are published or not. The documents may come from teaching and research institutions in France or abroad, or from public or private research centers.
L'archive ouverte pluridisciplinaire HAL, est destinée au dépôt et à la diffusion de documents scientifiques de niveau recherche, publiés ou non, émanant des établissements d'enseignement et de recherche français ou étrangers, des laboratoires publics ou privés. 
Titre

Evaluer la douleur en rhumatologie - comment faire mieux ? Une revue de la littérature

Auteurs: Florian Bailly ${ }^{1}$, Bruno Fautrel ${ }^{1}$, Laure Gossec ${ }^{1}$

Correspondance :

Dr Florian Bailly

Service de rhumatologie - Hôpital Pitié Salpêtrière

47-83 boulevards de l'hôpital

75013 Paris

Florian.bailly@psl.aphp.fr

0142177812

Institutions

Florian Bailly, MD, Laure Gossec, MD, PhD

Sorbonne Universités, UPMC Univ Paris 06, Institut Pierre Louis d'Epidémiologie et de Santé Publique; AP-HP, hôpital Pitié Salpêtrière, Service de rhumatologie, F75013, Paris, France

Financement : aucun

Conflits d'intérêt: aucun 


\section{Résumé}

La rhumatologie est une spécialité où l'on est confronté aux symptômes des patients, au premier rang desquels figure la douleur. Il existe plusieurs scores validés pour évaluer la douleur. Les échelles unidimensionnelles de la douleur, telles que l'échelle visuelle analogique ou l'échelle numérique simple sont rapides à réaliser mais présentent certaines limitations importantes à connaitre. Certaines situations cliniques nécessitent l'emploi de questionnaires multidimensionnels, telles que le Questionnaire Saint-Antoine ou 1'Inventaire multidimensionnel de la douleur par exemple. L'ensemble des composantes de la douleur peut ainsi être évaluée et prise en charge. Dans cet article, nous verrons les principaux critères rapportés par le patient permettant d'évaluer la douleur, et les propriétés psychométriques de ces questionnaires. Nous discuterons aussi du choix d'un questionnaire pour évaluer la douleur selon le cadre (pratique quotidienne, recherche).

\section{Mots clefs}

Douleur ; critères rapportés par les patients ; Questionnaire, Patient reported outcomes, Score 


\section{Introduction}

Dans le domaine des pathologies douloureuses, l'évaluation de la douleur est primordiale. La plupart des pathologies rhumatologiques sont responsables de douleurs. La douleur est donc à la fois un symptôme d'appel fréquent mais également un problème majeur dans le vécu de pathologies rhumatologiques chroniques. Ainsi dans la polyarthrite rhumatoïde, la douleur est l'élément clé dans l'impact pour le patient. L'évaluation de la douleur est essentielle pour le suivi d'un patient douloureux puisqu'elle joue un rôle clé dans les décisions thérapeutiques.

L'évaluation de la douleur dans son intensité mais aussi ses caractéristiques précises, localisation, circonstances déclenchantes est importante. L'intérêt se situe également pour la relation médecin malade puisqu'il s'agit d'être à l'écoute de ce symptôme si difficile à vivre pour les patients, et si difficile à traiter pour le praticien [1].

Au-delà de l'échange libre avec le patient, les questionnaires ou patient reported outcomes peuvent aider en consultation. Ils sont également importants à connaître pour interpréter les articles scientifiques.

Dans cet article, nous allons présenter une revue des outils utilisés pour évaluer la douleur en rhumatologie. Nous discuterons également de l'interprétation des scores et de l'évaluation des propriétés psychométriques des indices. Enfin nous discuterons du choix d'un outil selon la situation.

\section{Propriétés psychométriques des scores}

Un score ou questionnaire, pour être utilisable, doit être validé en termes de propriétés métrologiques. Ces propriétés ont été définies par le «filtre OMERACT » [2,3] qui a été mis au point par un groupe de méthodologistes (OMERACT, Outcome Measures in Rheumatology) et qui définit ces diverses propriétés métrologiques, appelées truth, feasibility, 
et discriminant capacity. Nous ferons référence à ces notions à propos des différents questionnaires de douleur - mais de quoi s'agit-il ?

\subsection{La validité}

La validité (truth) d'une échelle représente sa capacité à mesurer ce qu'elle est censée mesurer [2]. Il s’agit de la qualité métrologique la plus importante.

La validité d'apparence (face validity), fait référence à la validité de premier abord d'un questionnaire, ou acceptabilité subjective, estimée par les experts et/ou les patients selon un jugement subjectif sur l'impression générale du questionnaire (libellé et compréhension des questions, modalités des réponses et domaines explorés). La validité de contenu (content validity) fait référence à la pertinence des items, et à la capacité du critère à prendre en compte tous les attributs de la notion à mesurer. La validité de construit évalue la pertinence de la décomposition du critère en dimensions. Ceci évalue la cohérence interne : l'alpha de Cronbach (qui se score de 0 à 1). La validité de corrélation (concurrent validity) ou de critère (criterion validity) est évaluée par la corrélation entre le nouvel instrument et un outil qui a déjà fait ses preuves. On parle de validité de critère s'il s'agit de mesurer le critère contre un critère extérieur dit de référence ou 'gold standard'.

\subsection{La fiabilité}

La fiabilité (reliability) rend compte du degré d'erreur de mesure, ou capacité à donner des résultats comparables lorsque l'état est constant et que les situations expérimentales varient. Un instrument de mesure est fiable s'il produit les mêmes scores lors de mesures effectuées à différents moments par le même examinateur (reproductibilité inter-temps, test-retest reliability ou intra-rater reliability) et lors de mesures effectuées au même moment par différents examinateurs (reproductibilité inter-juges, inter-rater reliability). La fiabilité est souvent rapportée par le calcul du kappa, dont le résultat est considéré comme bon s'il est 
supérieur à 0,60 . On peut également rapporter la corrélation ou la corrélation intraclasse, dont le résultat va de 0 à 1 , où 1 indique une reproductibilité parfaite.

\subsection{La sensibilité au changement}

La sensibilité au changement est l'aptitude d'une échelle à mettre en évidence un changement du statut clinique, entre 2 situations ou entre 2 moments donnés. La sensibilité au changement capte la différence entre des mesures faites à deux temps différents, par exemple sous l'effet d'un traitement, tandis que la capacité discriminante distingue 2 états (par exemple malade versus sain). La capacité discriminante et la sensibilité au changement sont particulièrement importantes pour un critère de réponse thérapeutique et peuvent être mesurés par l'effet-taille (effect size), considéré comme important si > 0,80.

\subsection{La faisabilité}

La faisabilité, ou simplicité, fait référence à l'acceptabilité objective et englobe les notions de temps nécessaire à l'examen, des contraintes pour le patient, du coût et /ou de la disponibilité des appareils de mesure...Il s'agit d'une propriété métrologique «à part » car elle ne se mesure pas avec des statistiques, sauf pour le temps de passation et éventuellement le taux de valeurs absentes.

\section{Questionnaires, scores et échelles de la douleur}

Il existe de nombreux questionnaires permettant d'évaluer la douleur et pouvant s'appliquer à la rhumatologie. Cependant, nous nous limiterons à décrire ceux qui ont été validés en termes de propriétés métrologiques. On peut en dénombrer au moins neuf (Tableau 1). Nous allons en voir les caractéristiques principales. 


\subsection{Les échelles génériques unidimensionnelles}

Les échelles unidimensionnelles sont simples d'utilisation, permettent de faire facilement des mesures répétées pour mesurer la variation d'intensité de la douleur et l'efficacité des traitements antalgiques. On peut facilement définir un seuil absolu ou relatif. Néanmoins elles ne différentient pas les différentes composantes de la douleur.

\subsubsection{L'échelle visuelle analogique (EVA) douleur.}

L'EVA douleur est l'outil d'auto-évaluation probablement le plus fréquemment utilisé en pratique quotidienne. Elle mesure l'intensité de la douleur via une échelle horizontale ou verticale de $10 \mathrm{~cm}$ de long, bornée par «pas de douleur » et «douleur maximale imaginable » [4,5]. L'EVA douleur existe sous forme imprimée ou sous forme de réglette : le patient trace un trait ou positionne le curseur sur l'échelle et au verso le soignant lit la douleur à l'aide d'une graduation en millimètres. Le score est une variable numérique continue allant de 0 à 100 (correspondant aux millimètres mesurés sur l'échelle), il est parfois noté selon une échelle de 0 à 10 si l'échelle est mesurée en centimètres, ou par une graduation de couleur rouge. Pour éviter le choix d'une valeur préférée ou habituelle par le répondant, il est recommandé de ne pas mettre de nombre ou de mot sur une partie de l'échelle autre que les extrémités, et d'utiliser une présentation neutre [5]. Métrologie: L'EVA douleur a une corrélation de 0,70 à 0,75 avec une échelle verbale simple [6]. L'EVA douleur est sensible au changement [7]. La différence minimale cliniquement pertinente est entre $11 \mathrm{~mm}$ et $20 \mathrm{~mm}$ ou une diminution de $30 \%$ du score selon les pathologies [8-10]. 


\subsubsection{L'échelle numérique simple (ENS)}

L'ENS est une mesure unidimensionnelle de la douleur [11,12] dont plusieurs versions existent. La version la plus commune est une version segmentée issue de l'EVA douleur où le répondant choisit un nombre plein de 0 à 10 , reflétant l'intensité de la douleur. Comme pour l'EVA douleur les nombres sont bornés par «pas de douleur » et «pire douleur imaginable » [6,11,12]. Elle peut être administrée verbalement ou graphiquement [13], sur un trait horizontal. L'ENS est parfois préférée à une EVA douleur par des patients douloureux chroniques[14,15].

Métrologie : L'ENS est fortement corrélée $(0,86$ à 0,95$)$ à l'EVA douleur [6,16]. La différence minimale cliniquement significative est de 2 points ou une modification de $30 \%$ du score [10].

\subsubsection{L'échelle verbale simple}

L'échelle verbale simple correspond à une échelle de type Likert et propose une série de qualificatifs hiérarchisés pour coter sa douleur; aucune douleur, douleur faible, douleur moyenne, douleur sévère ou douleur très sévère [17]. Néanmoins il n'existe pas de traduction française validée et l'utilisation d'un nombre variable de seuils rend difficile son interprétation. Elle s'adresse principalement aux patients incapables d'utiliser une EVA ou une ENS (incompréhension, difficultés d'abstraction).

Métrologie : L'EVA, ENS et échelle verbale simple ont une corrélation de 0,70 à 0,75 $[18,19]$ mais elles se sont pas linéaires et ces échelles ne sont pas superposables. La variabilité de l'effet placebo selon les différents types d'échelles n'a pas non plus été évaluée.

\subsection{Les échelles multidimensionnelles}

Elles permettent de préciser la sémiologie de la douleur et de prendre en compte l'ensemble de ses composantes sensorielles et émotionnelles, mais sont plus longues à remplir et à scorer. 


\subsubsection{Le McGill Pain questionnaire}

Le McGill Pain Questionnaire (MPQ) [20] est un questionnaire en langue anglaise constitué par une liste de 78 qualificatifs répartis en 20 sous-classes (correspondant à un aspect de la douleur) regroupées en 4 classes : sensorielle ou sensori-discriminative, affective ou affectivo-émotionnelle, évaluative et diverse (sensori-affective). Il permet de connaître l'intensité de la douleur mais aussi de nombreuses autres caractéristiques ce qui est utile notamment dans la douleur chronique. Une deuxième partie est constituée de deux questions sur l'intensité et la temporalité de la douleur. Un score de 0 à 78 peut être calculé.

Métrologie : Une méta-analyse a trouvé que le score varie de 24 à 50\% du score maximal dans 51 études différentes [21]. Sa reproductibilité est bonne ainsi que sa sensibilité au changement (Tableau 1) [22,23]. Sa validité a été testée chez des patients présentant différentes douleurs articulaires, les mots sélectionnés étaient similaires en cas de pathologie identique et le nombre de mots sélectionnés était corrélé à une EVA douleur [24]. Néanmoins il nécessite un vocabulaire riche, et n'est pas adapté aux patients avec une faible alphabétisation. Il n'y a pas de seuil défini pour l'interprétation.

\subsubsection{Le Short-form McGill Pain Questionnaire}

Le Short-form McGill Pain Questionnaire (SF-MPQ) est la version courte du McGill Pain Questionnaire [25], composée de 15 mots issus de l'original (11 mots sensoriels, 4 mots affectifs), un item sur l'intensité de la douleur et une EVA douleur (Annexe 1). Chaque mot est noté sur une échelle d'intensité $(0=$ absent, $1=$ léger, $2=$ modéré, $3=$ sévère). Un score total de 60 points peut être calculé, sans seuil établi. Sa passation est plus rapide que le MPQ original (2 à 5 minutes versus 5 à 15 minutes).

Métrologie : la corrélation entre les deux formes était entre 0.67 et 0.87 sur deux populations différentes [26]. Sa consistance interne chez des patients fibromyalgiques ou avec une 
polyarthrite rhumatoïde était de 0.73 à 0.89 [26]. La sensibilité au changement et la validité de contenu sont variables selon les pathologies [27].

\subsubsection{Le Questionnaire Douleur Saint-Antoine}

C'est une adaptation française modifiée du McGill Pain Questionnaire [27]. Il comprend 61 qualificatifs répartis en 17 sous classes : 9 sensorielles, 7 affectives et 1 évaluative. Il dépend très largement du niveau socio-culturel et des possibilités d'expression verbale du sujet.

Métrologie : sa validité de contenu est similaire au MPQ original [28]. Une version courte a 15 items a également été développée et doit être évaluée [28] (Annexe 2).

4.2.4 L'inventaire multidimensionnel de la douleur (West Haven-Yale Multidimensional Pain Inventory)

L'inventaire multidimensionnel de la douleur est la traduction validée du Multidimensional Pain Inventory. Il est constitué de 49 items évaluant l'impact de la douleur chronique sur plusieurs aspects : l'expérience du patient avec la douleur, le soutien que le patient perçoit de son principal aidant (en général son conjoint), et les activités quotidiennes [29,30]. Trois catégories d'adaptation ont été proposées : dysfonctionnel, difficultés interpersonnelles et adaptatif [31].

Métrologie : elle semble satisfaisante (Tableau 1) [22,32,33].

\subsubsection{Le Short Form 36 Bodily Pain Scale}

Le Short Form 36 Bodily Pain Scale (SF36 BPS) est une des 8 sous-échelles du SF36, questionnaire générique de qualité de vie, traduit et validé en français, et soumis à copyright [34]. Les 2 items du SF36 BPS évaluent l'intensité de la douleur sur une échelle de Likert à 6 graduations et l'interférence de la douleur sur le travail sur une échelle à 5 graduations. Le score est calculé par une addition des deux échelles puis transformé sur une échelle de 0 à 100 .

Métrologie : les caractéristiques psychométriques du SF36 [35,36] sont bonnes, mais la souséchelle SF36 BPS n'a pas été évaluée individuellement. 


\subsubsection{Measure of Intermittent and Constant Osteoarthritis Pain}

Le Measure of Intermittent and Constant Osteoarthritis Pain (ICOAP) est un questionnaire multidimensionnel spécifique de la douleur arthrosique[37]. Il évalue l'intensité, la fréquence et l'impact de la douleur sur l'humeur, le sommeil et la qualité de vie, indépendamment de l'incapacité. Sa traduction française est validée [38]. Deux sous-scores sont calculés par les 11 items : la douleur constante et la douleur intermittente.

Métrologie : la validité de [39] et la sensibilité au changement ont été validées. Pour l'instant, le recul sur ce score est limité.

\section{Discussion}

Une évaluation exacte et appropriée de la douleur permet au clinicien une prise en charge adaptée et personnalisée du patient. Un accès facile aux outils d'évaluation en langue française est disponible sur le site internet de la Société Française d'Etude et de Traitement de la Douleur : http://www.sfetd-douleur.org/adulte [40].

Les échelles unidimensionnelles telles que l'EVA, l'ENS ou l'échelle verbale simple permettent une évaluation rapide et facile à scorer de l'intensité de la douleur. La validité de ces 3 échelles est comparable chez des patients instruits, mais la facilité d'administration et la sensibilité au changement orientent vers l'ENS [13]. En revanche, chez les personnes très jeunes, très âgées ou avec des troubles cognitifs, la réalisation de l'échelle verbale simple est préférée [41]. En recherche, l'ENS est préférée pour son administration possible par écrit ou oralement. Aucune de ces échelles unidimensionnelles ne permet une évaluation complète de la composante douloureuse; mais elles peuvent permettre des auto-évaluations répétées car elles sont très rapides à remplir. La réalisation d'une ENS régulière, par exemple hebdomadaire, entre 2 consultations, en particulier via les outils modernes de l'e-santé (sites 
internet etc) pourrait permettre de mieux appréhender le vécu douloureux d'un patient. Cependant à moyen terme, de nombreux patients peuvent ne pas vouloir s'autoévaluer régulièrement [42]. Il est également possible que des mesures trop répétées de la douleur puissent modifier celle-ci, en particulier dans le sens d'une majoration. Cette majoration pourrait être favorisée par la banalisation de mesures chiffrées itératives. La fréquence optimale de mesure de la douleur n'est pas connue à ce jour et pourrait être un axe de recherche pertinent.

Par ailleurs, certains patients peuvent changer leur comportement simplement parce qu'ils sont observés ou étudiés (effet Hawthorne), ce qui peut conduire à une réduction de la douleur [43]. Cet effet peut être influencé notamment par la personnalité de l'évaluateur.

Certains patients, lors d'une évaluation par une échelle visuelle ou numérique simple, rapportent une intensité à 10/10 dans un calme absolu, ce qui laisse souvent perplexe l'évaluateur. Il est probable que certains patients, devant « résumer » leur douleur sur une échelle unidimensionnelle, majorent l'intensité pour signifier différentes composantes ou conséquences de la douleur. Par exemple pour signifier sa durée, sa répercussion sur le moral ou le handicap. Ce type de réponse semblant « paradoxale » doit inciter le clinicien à utiliser une échelle multidimensionnelle plus adaptée à ce type de vécu.

Dans le cadre d'études centrées sur la douleur, les questionnaires multidimensionnels sont souvent préférés aux questionnaires uniques. Le McGill Pain Questionnaire, et son équivalent français le Questionnaire Saint-Antoine, sont utilisés largement en recherche pour mesurer non seulement l'intensité de la douleur mais aussi le type de douleur. Le SF36 et sa sous échelle BPS sont utiles pour mesurer la douleur dans le contexte général de la santé et qualité de vie, et permettent de réaliser des comparaisons entre différentes populations. A l'inverse du Questionnaire Saint-Antoine, le SF-36 BPS est suffisamment simple pour pouvoir éventuellement être réalisé en routine clinique. Les questionnaires spécifiques, comme 
l'ICOAP, permettent une évaluation fine pour une pathologie donnée à la fois en pratique clinique et surtout en recherche.

L'adaptation du questionnaire à l'état cognitif et intellectuel du patient est importante. Chez des patients non communicants, plusieurs options comme les échelles des patients non communicants DOLOPLUS ou ALGOPLUS [44], ou l'échelle des visages peuvent être utilisés. En revanche la mise en évidence de patients présentant des difficultés d'abstraction ou des troubles cognitifs débutants peut être difficile. Le problème est bien sûr commun à tous les critères rapportés par les patients. Une évaluation de 10 questionnaires rhumatologiques dans une population en Grande Bretagne a indiqué une difficulté de compréhension de l'ensemble des questionnaires pour 22\% de la population testée [45].

En conclusion, une évaluation formalisée de la douleur est une première étape nécessaire dans la prise en charge d'un patient douloureux. Dans cet article, nous avons présenté les principaux scores validés permettant d'évaluer la douleur et discuté du choix d'un score qui variera bien sûr selon la situation. Cette revue devrait permettre aux lecteurs de mieux appréhender l'évaluation de la douleur, pour la pratique quotidienne comme pour la recherche. 


\section{Bibliographie}

[1] Machado GC, Maher CG, Ferreira PH, Pinheiro MB, Lin C-WC, Day RO, et al. Efficacy and safety of paracetamol for spinal pain and osteoarthritis: systematic review and meta-analysis of randomised placebo controlled trials. BMJ 2015;350:h1225.

[2] Boers M, Brooks P, Strand C V, Tugwell P. The OMERACT filter for Outcome Measures in Rheumatology. J Rheumatol 1998;25:198-9.

[3] Kirwan JR, Bartlett SJ, Beaton DE, et al. Updating the OMERACT filter: implications for patient-reported outcomes. J Rheumatol 2014;41:1011-5.

[4] Huskisson E. Measurement of pain. Lancet 1974;304:1127-31.

[5] Scott J, Huskisson EC. Graphic representation of pain. Pain 1976;2:175-84.

[6] Downie WW, Leatham PA, Rhind VM, Wright V, Branco JA, Anderson JA. Studies with pain rating scales. Ann Rheum Dis 1978;37:378-81.

[7] Joyce CRB, Zutshi DW, Hrubes V, Mason RM. Comparison of fixed interval and visual analogue scales for rating chronic pain. Eur J Clin Pharmacol 1975;8:415-20.

[8] Tashjian RZ, Deloach J, Porucznik CA, Powell AP. Minimal clinically important differences (MCID) and patient acceptable symptomatic state (PASS) for visual analog scales (VAS) measuring pain in patients treated for rotator cuff disease. J Shoulder Elb Surg 2009;18:927-32. 
[9] Wolfe F, Michaud K. Assessment of pain in rheumatoid arthritis: Minimal clinically significant difference, predictors, and the effect of anti-tumor necrosis factor therapy. $\mathbf{J}$ Rheumatol 2007;34:1674-83.

[10] Farrar JT, Young JP, LaMoreaux L, Werth JL, Poole RM. Clinical importance of changes in chronic pain intensity measured on an 11-point numerical pain rating scale. Pain 2001;94:149-58.

[11] Childs JD, Piva SR, Fritz JM. Responsiveness of the numeric pain rating scale in patients with low back pain. Spine (Phila Pa 1976) 2005;30:1331-4.

[12] Jensen MP, McFarland CA. Increasing the reliability and validity of pain intensity measurement in chronic pain patients. Pain 1993;55:195-203.

[13] Jensen MP, Karoly P, Braver S. The Measurement of Clinical Pain Intensity: a Comparison of Six Methods. Pain 1986;27:117-26.

[14] Hush JM, Refshauge KM, Sullivan G, De Souza L, McAuley JH. Do numerical rating scales and the Roland-Morris Disability Questionnaire capture changes that are meaningful to patients with persistent back pain? Clin Rehabil 2010;24:648-57.

[15] Williams ACDC, Davies HTO, Chadury Y. Simple pain rating scales hide complex idiosyncratic meanings. Pain 2000;85:457-63.

[16] Ferraz MB, Quaresma MR, Aquino LRL, Atra E, Tugwell P, Goldsmith CH. Reliability of pain scales in the assessment of literate and illiterate patients with rheumatoid arthritis. J Rheumatol 1990;17:1022-4.

[17] Keele KD. The Pain Chart. Lancet 1948;252:6-8. 
[18] Reading AE. A comparison of pain rating scales. J Psychosom Res 1980;24:119-24.

[19] Atkinson JH, Ignelzi RJ, Jolla L. Measurement of pain : patient preference does not confound pain measurement. Pain 1981;10:241-8.

[20] Melzack R. The McGill pain questionnaire: major properties and scoring methods. Pain 1975;1:277-99.

[21] Wilkie DJ, Savedra MC, Holzemer WL, Tesler MD, Paul SM. Use of the McGill Pain Questionnaire to measure pain: a meta-analysis. Nurs Res 1990;39:36-41.

[22] Burckhardt CS, Jones KD. Adult measures of pain: The McGill Pain Questionnaire (MPQ), Rheumatoid Arthritis Pain Scale (RAPS), Short-Form McGill Pain Questionnaire (SF-MPQ), Verbal Descriptive Scale (VDS), Visual Analog Scale (VAS), and West Haven-Yale Multidisciplinary Pain Inven. Arthritis Rheum 2003;49:S96-104.

[23] Burckhardt CS. The use of the McGill Pain Questionnaire in assessing arthritis pain. Pain 1984;19:305-14.

[24] Papageorgiou AC, Badley EM. The quality of pain in arthritis: The words patients use to describe overall pain and pain in individual joints at rest and on movement. $\mathbf{J}$ Rheumatol 1989;16:106-12.

[25] Melzack R. The short-form McGill Pain Questionnaire. Pain 1987;30:191-7.

[26] Burckhardt CS, Bjelle A. A Swedish version of the short-form McGill Pain Questionnaire. Scand J Rheumatol 1994;23:77-81. 
[27] Boureau F, Luu M, Doubrere JF, Gay C. [Construction of a questionnaire for the selfevaluation of pain using a list of qualifiers. Comparison with Melzack's McGill Pain Questionnaire]. Therapie 1984;39:119-29.

[28] Boureau F, Luu M, Doubrère JF. Comparative study of the validity of four French McGill Pain Questionnaire (MPQ) versions. Pain 1992;50:59-65.

[29] Kerns RD, Turk DC, Rudy TE. The West Haven-Yale Multidimensional Pain Inventory (WHYMPI). Pain 1985;23:345-56.

[30] Laliberté S, Lamoureux J, Sullivan MJL, Miller JM, Charron J, Bouthillier D. French translation of the Multidimensional Pain Inventory: L'inventaire multidimensionnel de la douleur. Pain Res Manag 2008;13:497-505.

[31] Turk DC, Rudy TE. Toward an empirically derived taxonomy of chronic pain patients: integration of psychological assessment data. J Consult Clin Psychol 1988;56:233-8.

[32] Flavell HA, Carrafa GP, Thomas CH, Disler PB. Managing chronic back pain: impact of an interdisciplinary team approach. Med J Aust 1996;165:253-5.

[33] Kerns RD, Rosenberg R. Pain-relevant responses from significant others: Development of a significant-other version of the WHYMPI scales. Pain 1995;61:245-9.

[34] Leplège A, Ecosse E, Verdier A, Perneger T V. The French SF-36 Health Survey: translation, cultural adaptation and preliminary psychometric evaluation. J Clin Epidemiol 1998;51:1013-23. 
[35] Quintana JM, Escobar A, Bilbao A, Arostegui I, Lafuente I, Vidaurreta I. Responsiveness and clinically important differences for the WOMAC and SF-36 after hip joint replacement. Osteoarthritis Cartilage 2005;13:1076-83.

[36] Escobar A, Quintana JM, Bilbao A, Aróstegui I, Lafuente I, Vidaurreta I. Responsiveness and clinically important differences for the WOMAC and SF-36 after total knee replacement. Osteoarthritis Cartilage 2007;15:273-80.

[37] Hawker G a., Davis a. M, French MR, al. Development and preliminary psychometric testing of a new OA pain measure - an OARSI/OMERACT initiative. Osteoarthr Cartil 2008;16:409-14.

[38] Maillefert JF, Kloppenburg M, Fernandes L, et al. Multi-language translation and cross-cultural adaptation of the OARSI/OMERACT measure of intermittent and constant osteoarthritis pain (ICOAP). Osteoarthritis Cartilage 2009;17:1293-6.

[39] Hawker GA, Davis AM, French MR, et al. Development and preliminary psychometric testing of a new OA pain measure--an OARSI/OMERACT initiative. Osteoarthritis Cartilage 2008;16:409-14.

[40] http://www.sfetd-douleur.org/adulte . Société Française d'Etude et du Traitement de la Douleur. Date d'accès : 15/06/2015

[41] Herr KA, Spratt K, Mobily PR, Richardson G. Pain intensity assessment in older adults: use of experimental pain to compare psychometric properties and usability of selected pain scales with younger adults. Clin J Pain 2004;20:207-19. 
[42] Koevoets R, de Glas N A, le Bourlout C, et al. Autonomous online health assessment questionnaire registry in daily clinical practice. Rheumatology (Oxford) 2013;52:8837.

[43] Turner JA, Deyo RA, Loeser JD, Von Korff M, Fordyce WE. The importance of placebo effects in pain treatment and research. JAMA 1994;271:1609-14.

[44] Rat P, Jouve E, Pickering G, et al. Validation of an acute pain-behavior scale for older persons with inability to communicate verbally: Algoplus®. Eur J Pain 2011;15.

[45] Adams J, Chapman J, Bradley S, Ryan SJ. Literacy levels required to complete routinely used patient-reported outcome measures in rheumatology. Rheumatology (Oxford) 2013;52:460-4.

[46] Broderick JE, Schwartz JE, Vikingstad G, Pribbernow M, Grossman S, Stone AA. The accuracy of pain and fatigue items across different reporting periods. Pain 2008;139:146-57.

[47] Strand LI, Ljunggren AE, Bogen B, Ask T, Johnsen TB. The Short-Form McGill Pain Questionnaire as an outcome measure: Test-retest reliability and responsiveness to change. Eur J Pain 2008;12:917-25.

[48] Brazier JE, Harper R, Jones NM, et al. Validating the SF-36 health survey questionnaire: new outcome measure for primary care. BMJ 1992;305:160-4.

[49] Bombardier C, Melfi CA, Paul J, et al. Comparison of a generic and a disease-specific measure of pain and physical function after knee replacement surgery. Med Care 1995;33:AS131-S144. 


\begin{tabular}{|c|c|c|c|c|}
\hline Questionnaire & $\begin{array}{l}\text { Nombre de } \\
\text { questions }\end{array}$ & $\begin{array}{l}\text { Temps de } \\
\text { passage }\end{array}$ & $\begin{array}{l}\text { Validation en } \\
\text { français ? }\end{array}$ & Reproductibilité \\
\hline $\begin{array}{l}\text { Echelle visuelle } \\
\text { analogique douleur }\end{array}$ & 1 & $<1$ minute & Oui & $\begin{array}{l}\mathrm{R}=0,93 \text { chez les } \\
\text { patients instruits, } \\
\mathrm{r}=0,71 \text { chez des } \\
\text { patients illettrés [16] }\end{array}$ \\
\hline $\begin{array}{l}\text { Echelle numérique } \\
\text { simple }\end{array}$ & 1 & $<1$ minute & N/A & $\mathrm{R}=0,95[16]$ \\
\hline $\begin{array}{l}\text { Echelle verbale } \\
\text { simple }\end{array}$ & 1 & $<1$ minute & Non validé & $\mathrm{R}=0,82[16]$ \\
\hline $\begin{array}{l}\text { McGill pain } \\
\text { questionnaire }\end{array}$ & 79 items & $5-10$ minutes & Non validé & $\mathrm{R}=0,59-0,81[46]$ \\
\hline $\begin{array}{l}\text { Short-form McGill } \\
\text { Pain Questionnaire }\end{array}$ & 15 items & $2-5$ minutes & Non validé & $\mathrm{R}=0,75$ à 0,93 [47] \\
\hline $\begin{array}{l}\text { Questionnaire } \\
\text { Douleur Saint- } \\
\text { Antoine }\end{array}$ & 61 items & Non évalué & Validé & Non évalué \\
\hline $\begin{array}{l}\text { L'inventaire } \\
\text { multidimensionnel } \\
\text { de la douleur }\end{array}$ & 49 items & 5-10 minutes & Validé & $\mathrm{R}=0,62-0,91[22]$ \\
\hline $\begin{array}{l}\text { Le Short Form } 36 \\
\text { Bodily Pain Scale }\end{array}$ & 2 items & $<2$ minutes & Validé & $\mathrm{R}=0,78[48]$ \\
\hline Measure & 11 items & $<10$ minutes & Validé & $\mathrm{R}=0,85$ [49] \\
\hline
\end{tabular}




\begin{tabular}{|l|l|l|l|}
\hline Intermittent and & & & \\
Constant & & & \\
Osteoarthritis Pain & & & \\
\hline
\end{tabular}

Tableau 1: Résumé des caractéristiques des principaux questionnaires douleur: nombre d'items, temps de passation, validation en français, reproductibilité. 


\section{Annexes}

\subsection{Annexe 1 : Short Form Mc Gill Pain Questionnaire}

\section{SHORT-FORM MEGILL PAIN QUESTIONNAIRE}

RONALD MELZACK

PATIENTS NAME:

DATE:

\begin{tabular}{|c|c|c|c|c|}
\hline & NONE & MILLD & MODERATE & SEVERE \\
\hline THROBBING & 0) & 1). & 2) & 3) \\
\hline SHOOTING & 0) & 1) & 2) & 3) _ \\
\hline STABBING & 0) & 1) & 2) & 3) \\
\hline SHARP & 0) & 1) & 2) & 3) \\
\hline CRAMPING & 0) & 1) & 2) & 3) _ _ _ - \\
\hline GNAWING & 0) & 1) & 2) & 3) \\
\hline HOT-BURNING & 0) & 1) & 2) & 3) \\
\hline ACHING & 0). & 1) & 2) & 3) \\
\hline HEAVY & 0) & 1) & 2) & 3) \\
\hline TENDER & 0) & 1) & 2) & 3) \\
\hline SPLITTING & 0) & 1) ... & 2) & 3) \\
\hline TIRING-EXHAUSTING & 0) & 1) & 2) & 3) \\
\hline SICKENING & 0) $\ldots$ & 1) & 2) & 3) \\
\hline FEARFUL & 0) & 1) & 2) & 3) \\
\hline PUNISHING-CRUEL & 0) & 1) ___..... & 2) & 3) \\
\hline
\end{tabular}

WORST

No POSSIBLE PAIN 1 PAIN

P P I

- NO PAIN

1 MILD

2 DISCOMFORTING

3 DistaEssing

4 HORRIBLE

5 EXCRUCIATING

(C) R. Melzack, 1984 


\subsection{Annexe 2 : Questionnaire Saint-Antoine abrégé}

\begin{tabular}{|c|c|c|c|c|c|}
\hline & $\begin{array}{c}0 \\
\text { absent } \\
\text { non }\end{array}$ & $\begin{array}{c}1 \\
\text { faible }\end{array}$ & $\begin{array}{c}2 \\
\text { modéré } \\
\text { modérément }\end{array}$ & $\begin{array}{c}3 \\
\text { fort } \\
\text { beaucoup }\end{array}$ & $\begin{array}{c}4 \\
\text { extrêmement } \\
\text { fort } \\
\text { extrêmement }\end{array}$ \\
\hline \multicolumn{6}{|l|}{ Élancements } \\
\hline \multicolumn{6}{|l|}{ Pénétrante } \\
\hline \multicolumn{6}{|c|}{ Décharges électriques } \\
\hline \multicolumn{6}{|c|}{ Coups de poignard } \\
\hline \multicolumn{6}{|c|}{ En étau } \\
\hline \multicolumn{6}{|l|}{ Tiraillement } \\
\hline \multicolumn{6}{|l|}{ Brûlure } \\
\hline \multicolumn{6}{|c|}{ Fourmillements } \\
\hline \multicolumn{6}{|c|}{ Lourdeur } \\
\hline \multicolumn{6}{|l|}{ Épuisante } \\
\hline \multicolumn{6}{|l|}{ Angoissante } \\
\hline \multicolumn{6}{|l|}{ Obsédante } \\
\hline \multicolumn{6}{|l|}{ Insupportable } \\
\hline \multicolumn{6}{|l|}{ Énervante } \\
\hline \multicolumn{6}{|l|}{ Exaspérante } \\
\hline Déprimante & & & & & \\
\hline
\end{tabular}

